\title{
Innovation
}

\section{Constant angular velocity of the wrist during the lifting of a sphere}

\author{
P. H. CHAPPELL*†, C. D. METCALF† J. H. BURRIDGE,+ V. T. YULE $\$$ and \\ R. M. PICKERING $\S$
$†$ School of Electronics and Computer Science, University of Southampton, Highfield, Southampton SO17 1BJ, UK
†School of Health Sciences, University of Southampton, Highfield, Southampton SO17 1BJ, UK $\S$ School of Medicine, University of Southampton, Highfield, Southampton SO17 1BJ, UK

(Received 17 December 2009; revised 21 January 2010; accepted 29 January 2010)

\begin{abstract}
The primary objective of the experiments was to investigate the wrist motion of a person while they were carrying out a prehensile task from a clinical hand function test. A sixcamera movement system was used to observe the wrist motion of 10 participants. A very light sphere and a heavy sphere were used in the experiments to study any mass effects. While seated at a table, a participant moved a sphere over a small obstacle using their dominant hand. The participants were observed to move their wrist at a constant angular velocity. This phenomenon has not been reported previously. Theoretically, the muscles of the wrist provide an impulse of force at the start of the rotation while the forearm maintains a constant vertical force on a sphere. Light-heavy mean differences for the velocities, absolute velocities, angles and times taken showed no significant differences $(p=0.05)$.
\end{abstract}

Keywords: Wrist; Movement; Constant velocity; Projectile; SHAP

\section{Introduction}

Clinical gait analysis is an established method of quantifying the movement of the lower limbs and ambulatory patterns [1,2]. A common method used to capture data from gait is where reflective markers are located on the skin surface and a system of infrared cameras is used to determine the position and relative movement of limb segments. The extension of these techniques to the upper limb has not received the same attention due to the complexity of the anatomy and physiology where there is a wide range of different movement patterns [3-5].

Three-dimensional movement analysis has been applied to human research in a number of areas from rehabilitation to sports science. A trend developed recently in biomechanical research is to apply the principles established by the analysis of gait to the upper limb [6-11]. The motivation for using three-dimensional kinematic data originated from a desire to understand the biomechanical movements involved in prehensile tasks [12] and has evolved into developing an assessment for rehabilitation [4,13]. Understanding the variability associated with a particular movement in unimpaired participants brings greater understanding of the limitations and compensatory mechanisms involved in comparable movements by impaired participants. In addition, the current research trend is to concentrate on movements that are confined within defined and specific activities, whereby the movements generated by the upper limb are repetitive and constrained.

In this paper are presented experimental results from an investigation into the movement of the wrist. These results have been obtained from kinematic data while a person

*Corresponding author. Email: phc@ecs.soton.ac.uk 
carried out a prehensile task from a clinical hand function test. An objective of this research was to describe unimpaired functional movement of the wrist. Each participant completed a Southampton Hand Assessment Procedure (SHAP) [14]. Reflective markers were placed on the dominant upper limb of the participant, who was then instructed to complete five SHAP abstract object tasks, both light and heavyweight versions, while movements were captured by a Vicon movement analysis system $[15,16]$. Movements of the wrist were studied for light and heavyweight spherical, tripod, power, tip and extension abstract object tasks. However this paper focuses on the picking and placing of the spherical objects with different masses, in a cyclic movement (figure 1).

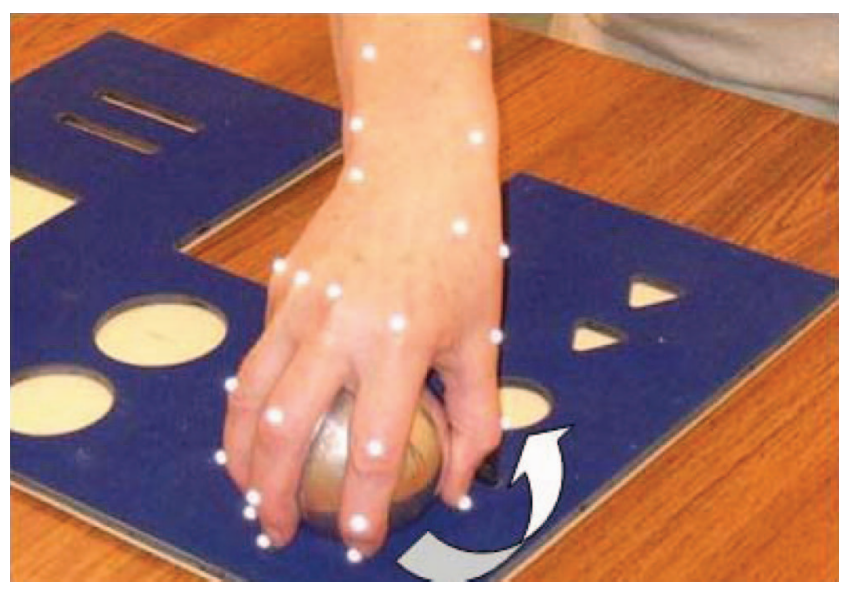

Figure 1. The reflective marker placements on the forearm and dorsal surface of a hand while moving a sphere over a hurdle.

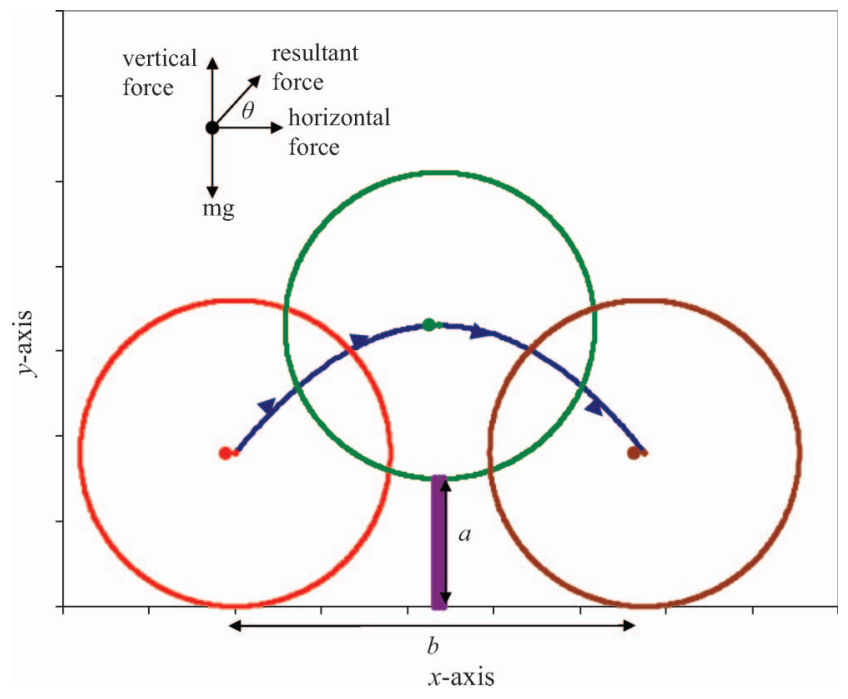

Figure 2. Side view of a sphere moving from left to right over a hurdle.

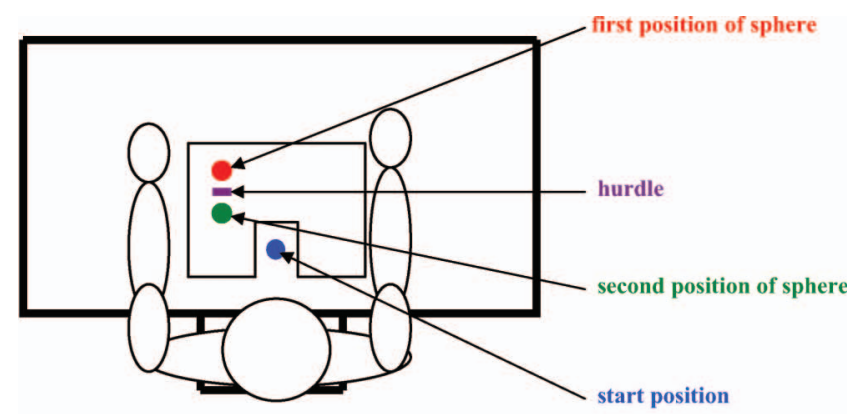

Plan view

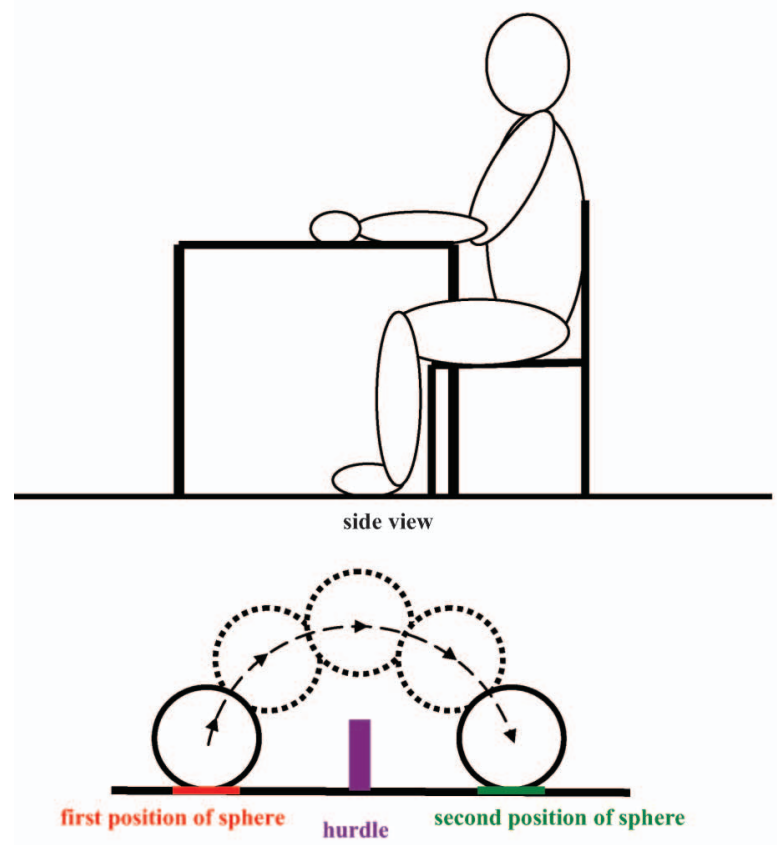

Side view of sphere movement

Figure 3. Diagrammatic drawings of the experimental layout.

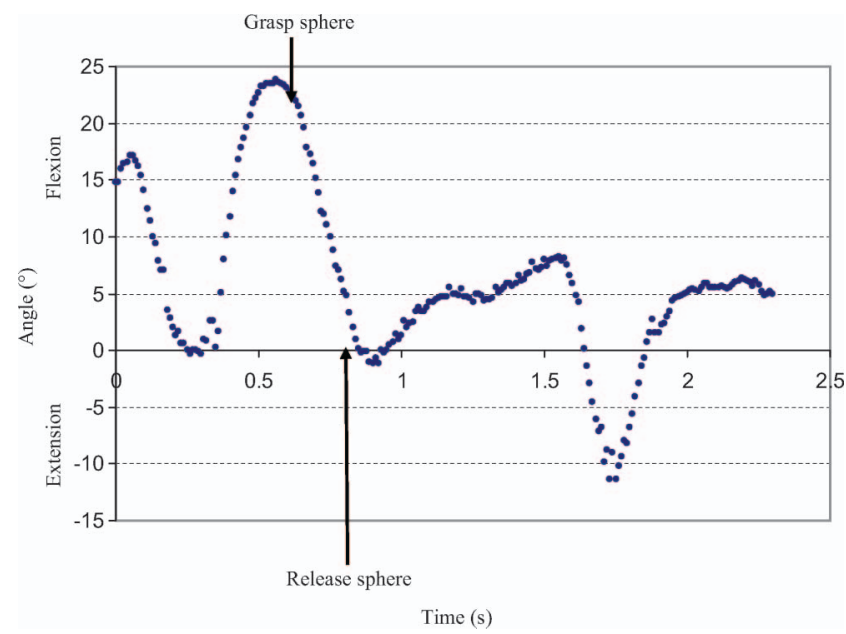

Figure 4. Waveform of participant 9 whilst lifting an aluminium sphere and extending their wrist (between the arrows). 
Table 1. Demographic data of trial participants, mean age $=33, \pm 9$ years.

\begin{tabular}{|c|c|c|c|c|c|c|c|}
\hline sex & handed & $18-25$ & $26-35$ & $36-45$ & $46-55$ & $56-65$ & total \\
\hline \multirow{5}{*}{$\begin{array}{l}\text { male } \\
\text { female } \\
\text { male } \\
\text { female }\end{array}$} & right & 1 & 1 & 1 & & & 3 \\
\hline & right & & 3 & 1 & 1 & & 5 \\
\hline & left & 1 & 1 & & & & 2 \\
\hline & left & & & & & & 0 \\
\hline & total & 2 & 5 & 2 & 1 & 0 & 10 \\
\hline
\end{tabular}

Table 2. Comparison of means between light and heavy spheres.

\begin{tabular}{|c|c|c|c|c|}
\hline & \multicolumn{2}{|c|}{ mean $(\mathrm{SD})$} & \multirow[b]{2}{*}{ light-heavy mean differences $(95 \% \mathrm{CI})$} & \multirow[b]{2}{*}{$p$} \\
\hline & light & heavy & & \\
\hline velocity, deg s ${ }^{-1}$ & $-11.3(44.3)$ & $-16.7(34.7)$ & $5.4(-16.1,26.9)$ & 0.582 \\
\hline absolute velocity, deg $\mathrm{s}^{-1}$ & 39.7 (18.9) & $34.0(20.5)$ & $5.7(-9.9,21.3)$ & 0.430 \\
\hline angle, deg & $-4.8(10.9)$ & $-6.3(11.8)$ & $1.5(-3.1,6.1)$ & 0.471 \\
\hline time, s & $0.31(0.07)$ & $0.36(0.1)$ & $-0.06(-0.11,0)$ & 0.066 \\
\hline
\end{tabular}

Participant 1
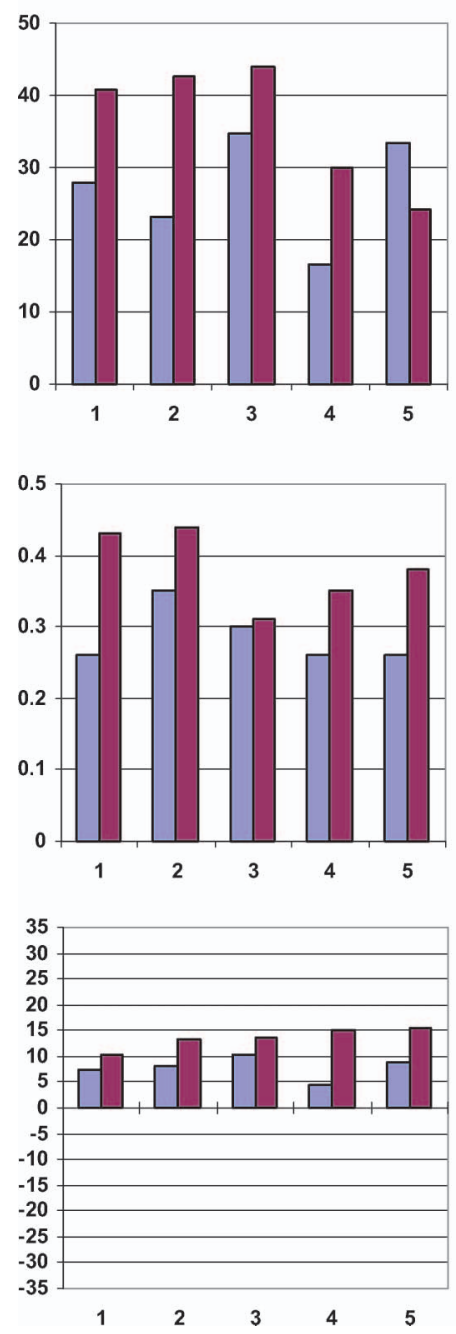

Participant 2
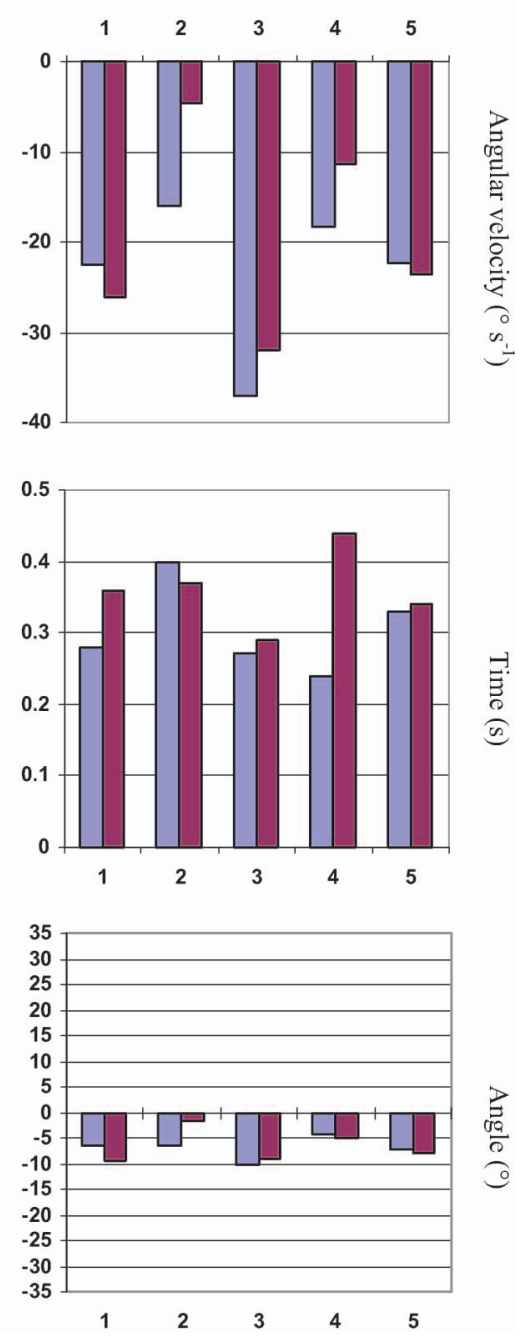

Figure 5. Angular velocities, times and angles for 10 subjects with five repeats. The left bar of each category is for a movement with the balsa sphere (blue) and to the right (purple) is with the aluminium sphere. Flexion of the wrist is indicated by positive values and negative values represent an extension. Note that 'angle' means the angle subtended during constant velocity. 
Participant 3
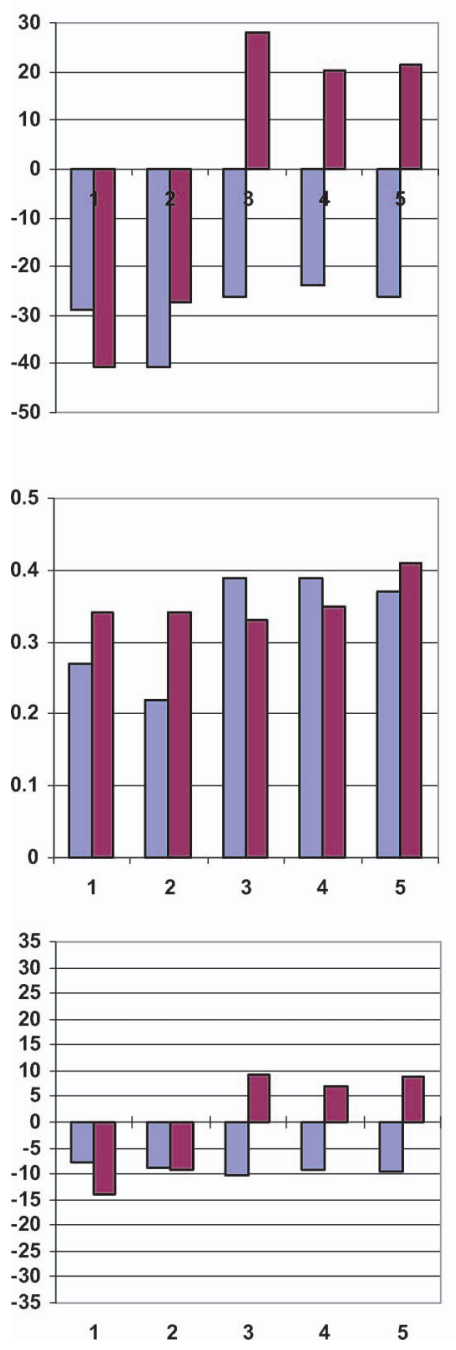

Participant 4
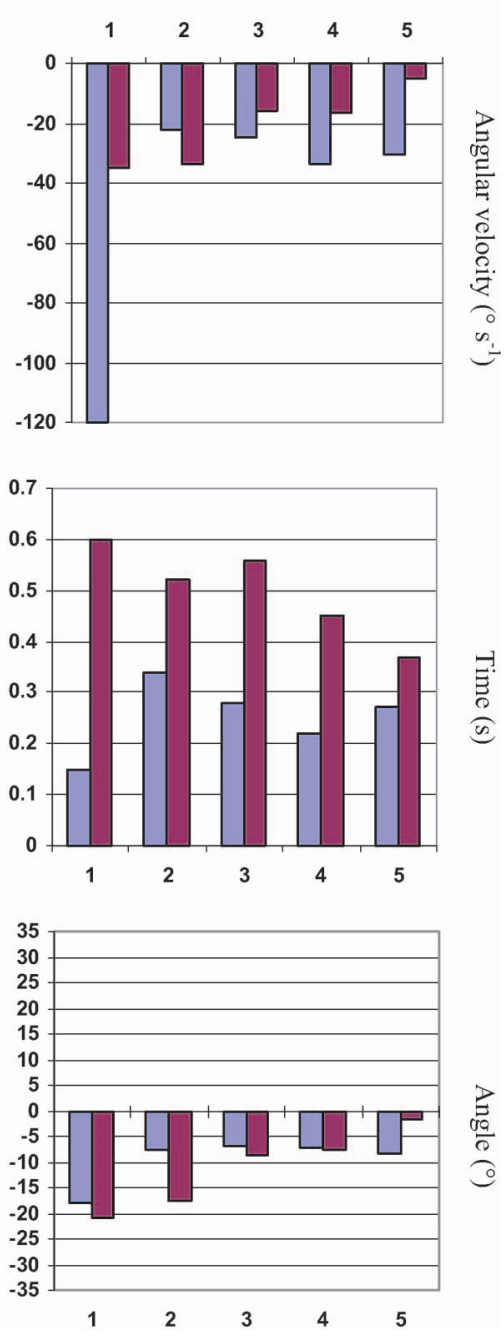

Figure 5b. (Continued).

There is evidence in the literature of the smooth operation of the wrist. Results of captured time series, from different experiments, show ramps of the wrist angle which appear to be linear [17-21]. These waveforms suggest that the wrist flexes and extends with a constant angular velocity, but there have been no experimental or theoretical reports of this phenomenon in the literature. This paper presents a retrospective analysis of experimental data to show for the first time that the wrist is moved with a constant angular velocity.

\section{Theory}

A three-dimensional model of the movement of the wrist and forearm is not presented in this paper. Instead, a two dimensional theory is given for the forces on a sphere that is moved in the prescribed manner shown in figure 1. From this theory and the experimental results a movement strategy can be inferred.

Consider a solid sphere that is held by a hand and being moved over a hurdle shown in figure 2. Generally, there will be forces and torques acting on the sphere from the wrist and forearm. The fingers, thumb and structures of the hand hold the sphere at the end of the forearm. Also the sphere is subjected to the Earth's gravitational field. More simply, for a sphere to be lifted from a surface and moved over the hurdle there will be vertical, horizontal and gravitational forces shown in the figure. This is a classical projectile problem where the motion has a parabolic path. Using a rectangular set of $x-y$ axes, the horizontal and vertical motions are as follows.

$$
x=u t \cos \theta
$$



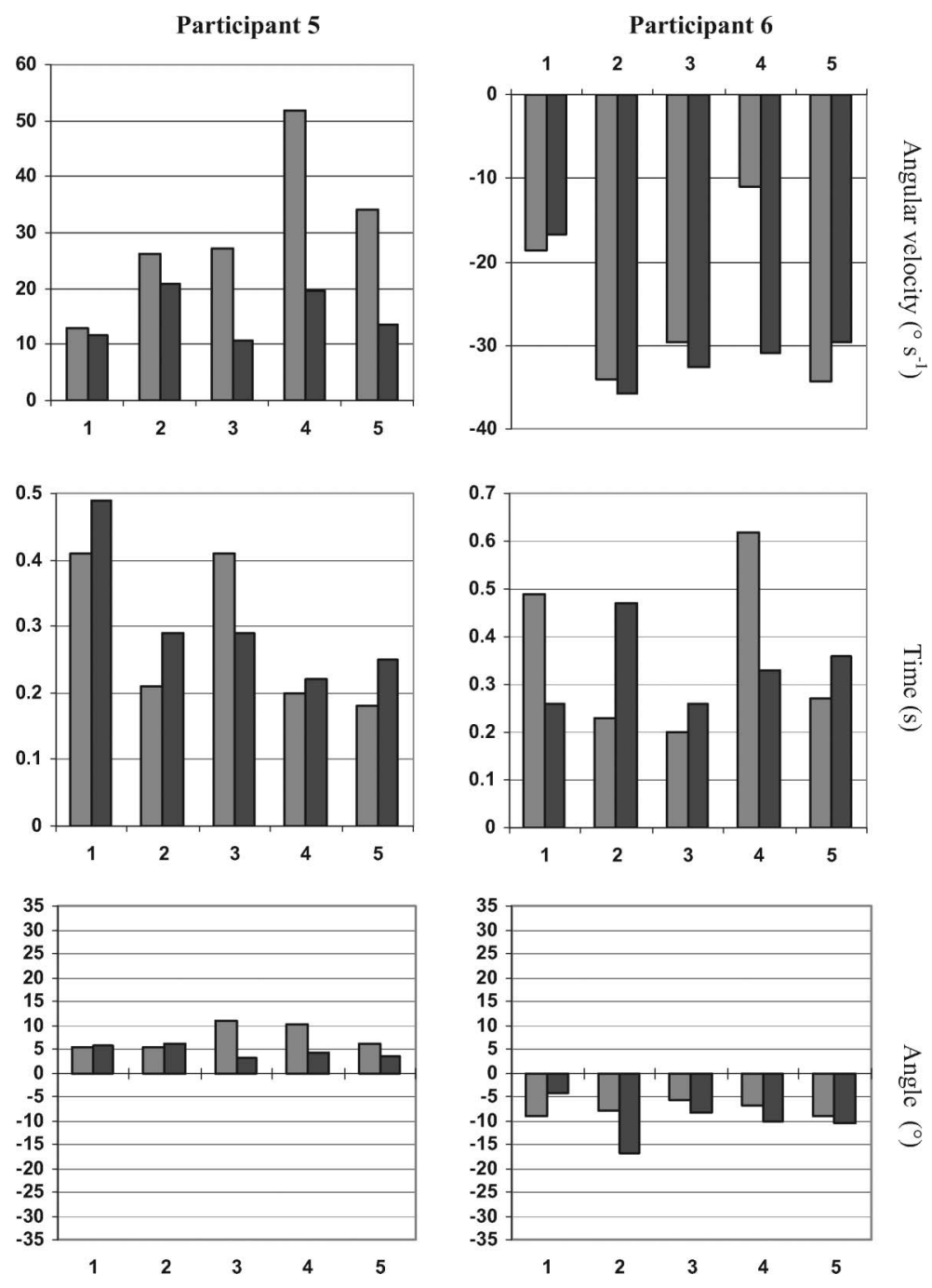

Figure 5c. (Continued).

where $u$ is the initial velocity at an angle $\theta$ to the horizontal $x$-axis and $t$ is the time.

$$
y=u t \sin \theta-\frac{1}{2} g t^{2},
$$

where $g$ is the acceleration due to gravity. The maximum altitude, $a$, is determined from these two equations, as:

$$
a=\frac{u^{2} \sin ^{2} \theta}{2 g} .
$$

The horizontal distance travelled, $b$, is given by:

$$
b=\frac{u^{2} \sin 2 \theta}{2 g} \text {. }
$$

This analysis is applicable where an impulse force has been applied by the upper limb to the projectile at the start and no other external forces are applied during the motion.

\section{Experiment}

The main aim of the experiment was to obtain waveforms of the wrist angle and investigate common features in them. A lightweight balsa sphere was used as it effectively represents the holding of an object with negligible mass. A heavyweight sphere, made from aluminium, with the same dimensions as the balsa sphere was also used.

A participant was seated at a table with their forearms resting on the table surface (figure 3). The sphere and hurdle were located in a form board on the table surface by the assessor as shown in figure 3. Using their dominant hand they were asked to carry out the following sequence of movements.

(1) touch the table surface in front of them (start position in the top drawing of figure 3);

(2) move to pick up the sphere (first position); 
Participant 7
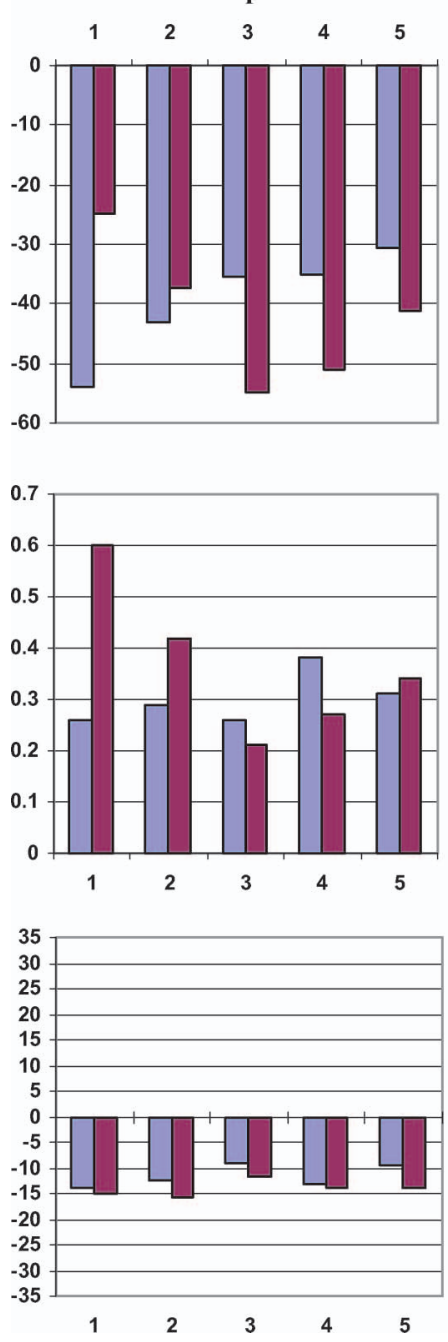

Participant 8
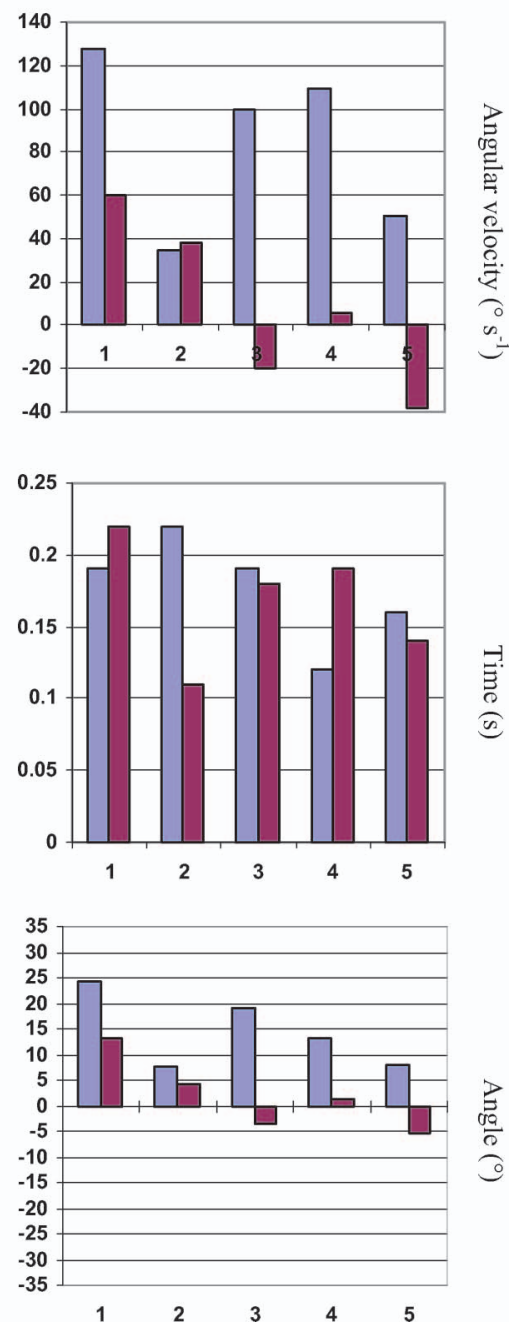

Figure 5d. (Continued).

(3) take the sphere over a $30 \mathrm{~mm}$ hurdle and place it down on the table surface (second position);

(4) return their hand to the start position; and

(5) place their forearm back on the table.

An assessor demonstrated the task to the participant and then asked them to carry out the movement as quickly as possible.

Ten unimpaired participants (mean age 33 years, \pm 9 years) were recruited for the trial with the following inclusion criteria:

- no history of musculoskeletal injury;

- no history of chronic pain in the dominant upper limb; and

- no present discomfort in their dominant upper limb.

Ethical approval for the trial was granted by the School of Health Professions \& Rehabilitation Sciences Ethical
Committee (submission number PO4-1101) at the University of Southampton. The demographic data of the participants is given in table 1 .

A six-camera movement analysis system (Vicon 460) was used to capture data at a sampling rate of $100 \mathrm{~Hz}$. A total of 26 hemispherical markers $(3 \mathrm{~mm})$ were placed on the hand and wrist $[15,16]$. The captured data were analysed to extract the flexion/extension angle of the wrist using Matlab software (MathWorks, Natick, MA, USA) [16]. A participant was asked to do five repeated movements for the balsa and aluminium spheres.

\section{Results}

From a waveform (time series) of the angle of the wrist, a ramp region was identified when a subject moved a sphere over the hurdle (figure 4). The data from the wrist angles were identified and cropped from the moment the spherical object was lifted from the table surface to the moment it 

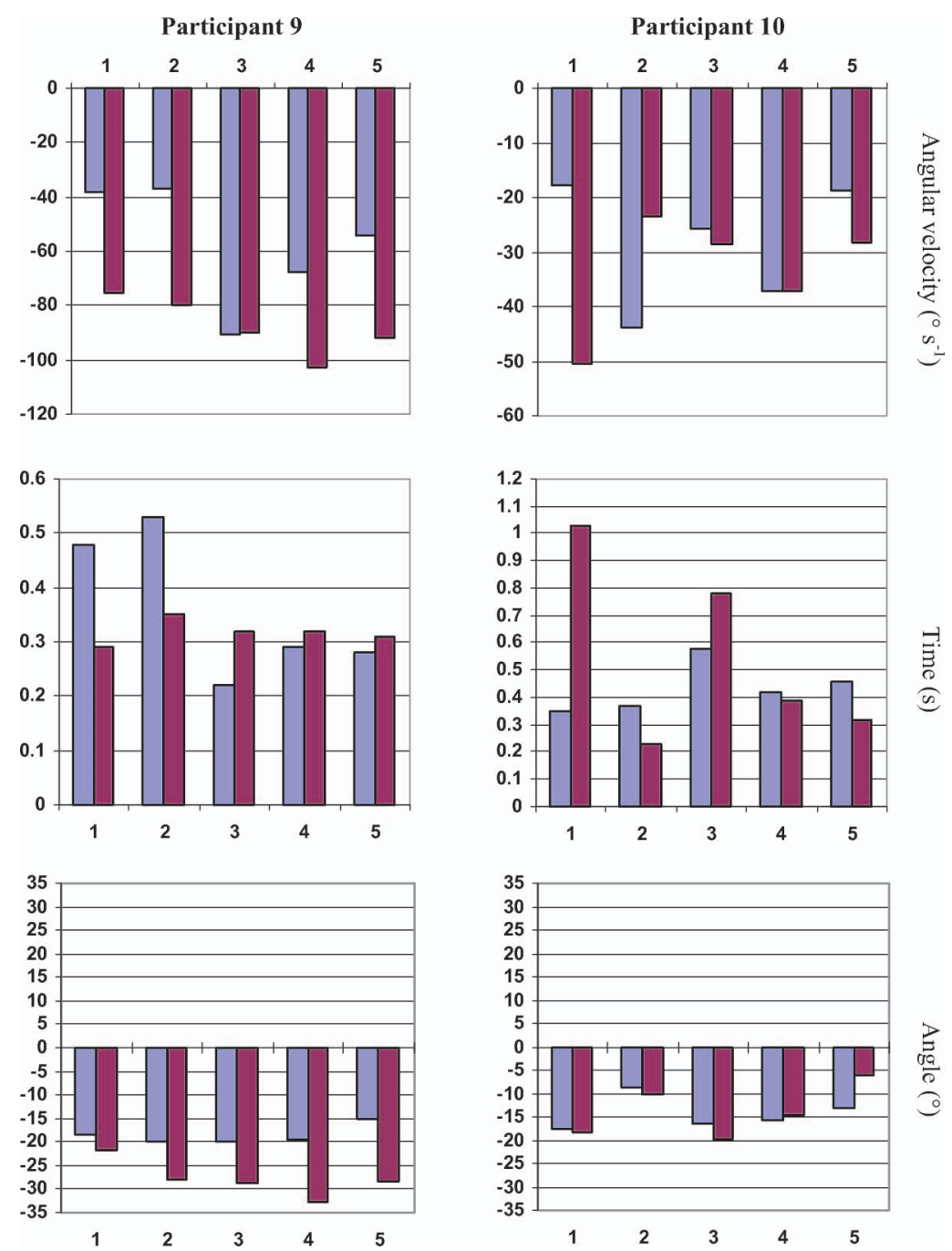

Figure 5e. (Continued).

was placed back on the table. An estimate of the slope (angular velocity) of a waveform for these linear regions was made using regression (mean $r^{2}$ for the task using the balsa sphere was 0.94 and that for the task using the aluminium sphere was 0.90 ). These values are near to unity which supports the hypothesis that the wrist movement is carried out with constant velocity.

Figure 5 shows the estimated angular velocities, times and angle for the 10 subjects. Six of the participants used increasing extension in the movement, while two used flexion and two a mixture of extension and flexion.

\subsection{Comparison of means between light and heavy weight spheres}

In table 2 the means for each variable across the five repeats under each condition have been calculated and compared between the light and heavy spheres in paired $t$-tests. Lightheavy mean differences are presented with $95 \%$ confidence intervals in the third column and in the last column the $p$ values show that the velocity, absolute velocity and angle have no significant differences. There was a trend of longer times to move the heavy sphere but this did not quite reach statistical significance $(p=0.066)$. Similar significance levels were obtained from non-parametric Wilcoxon signed rank tests with time again nearly reaching statistical significance $(p=0.059)$. Changes in velocity per participant are shown in figure 6 , where half of the participants have higher mean velocities with the heavy sphere and half lower. Four of the participants had a higher absolute angular velocity while moving the heavy sphere. Angular changes are shown in figure 7, where six of the participants have a higher mean with the heavy sphere. The trend of taking a longer time to move the heavy sphere, as seen in table 2, is shown in figure 8, where seven of the participants take a longer time, but there is not strong enough evidence to achieve statistical significance. 


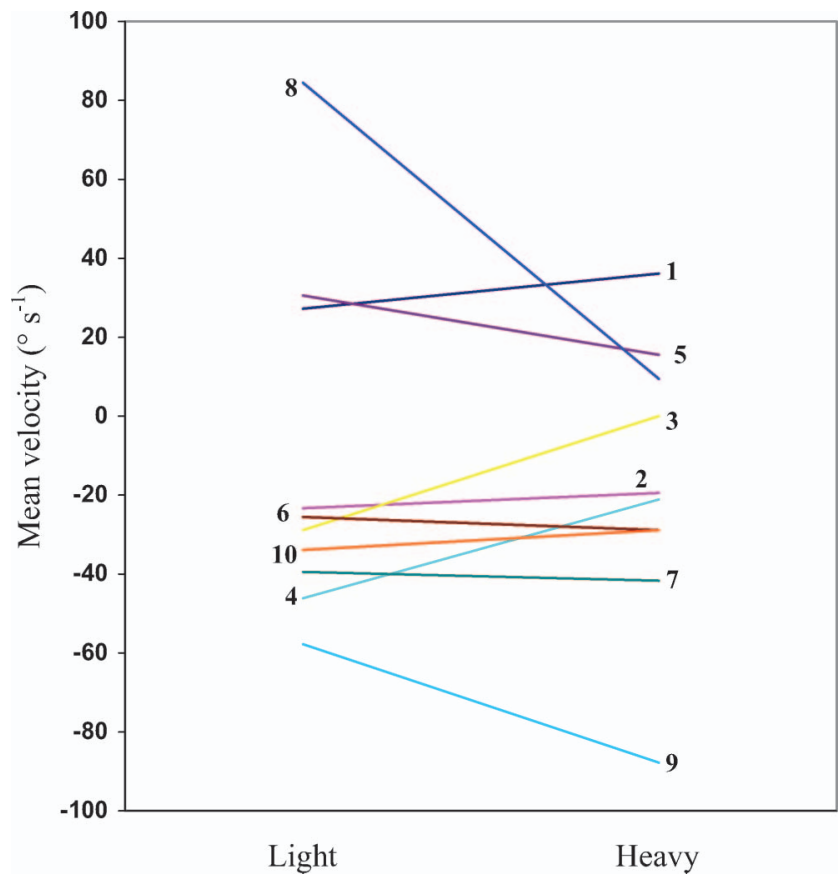

Figure 6. Mean velocity changes per participant.

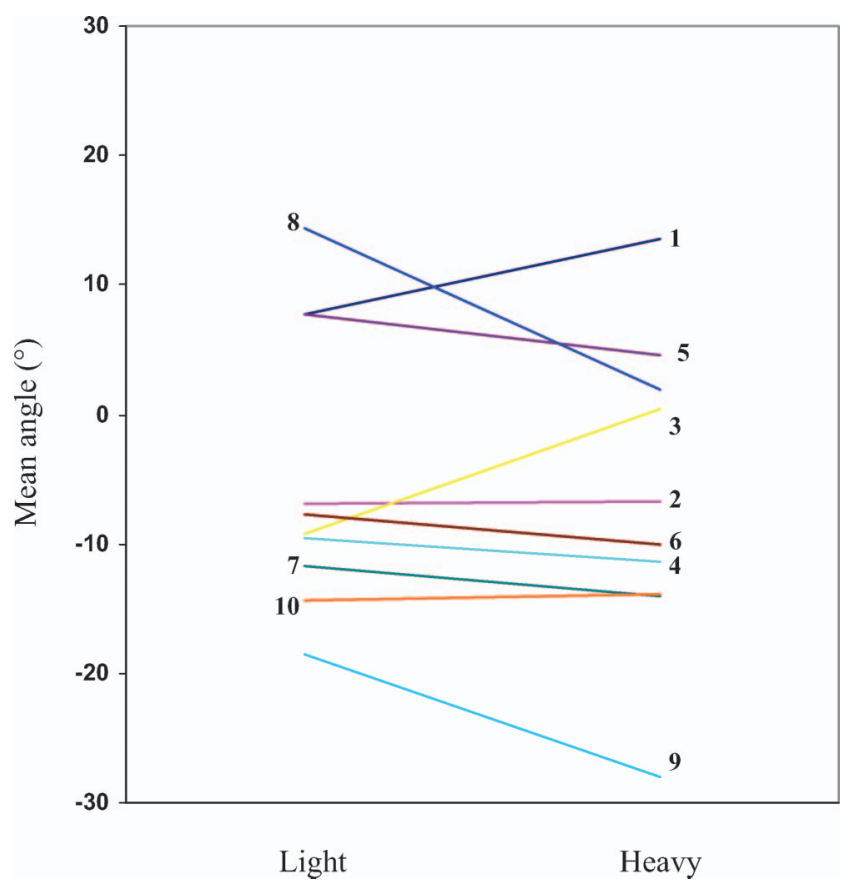

Figure 7. Mean angle changes per participant.

\section{Discussion}

Typically, the wrist was extended as a sphere was moved over the hurdle (figure 9). During the lifting of the sphere from the table surface to the top of the hurdle, the wrist provides vertical movement and little horizontal move-

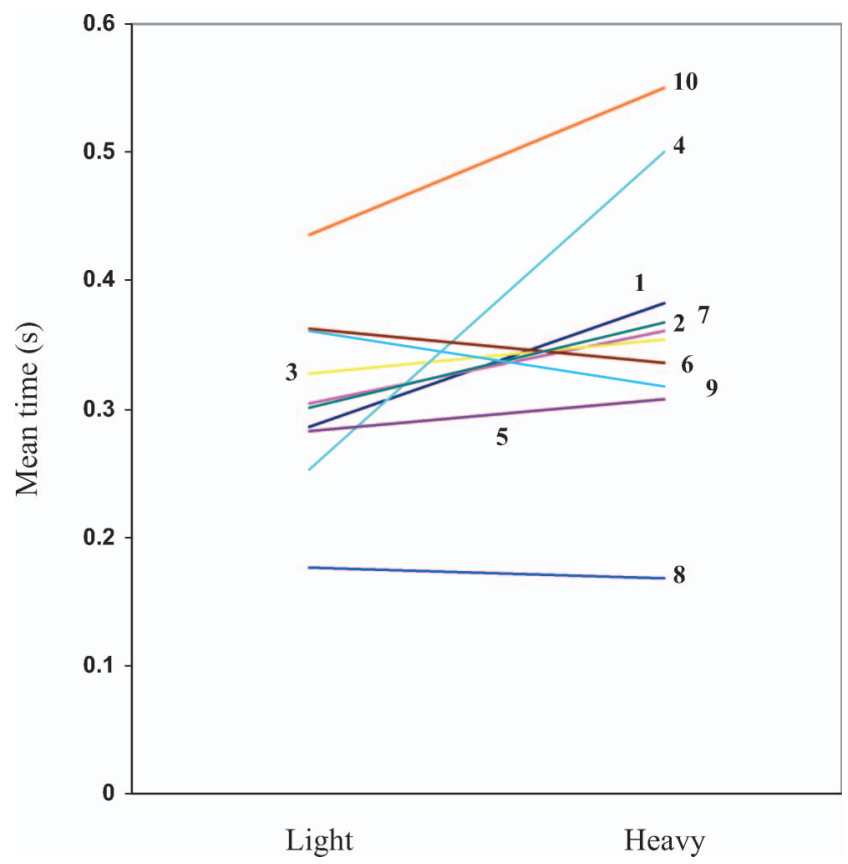

Figure 8. Mean time changes per participant.

ment. The latter is provided by the forearm and shoulder. For the sphere to land at the end position from the top of the hurdle onto the table, the forearm is lowered while the wrist extends, otherwise the sphere would continue to move upwards. There is a coordinated movement of the wrist maintaining a constant angular velocity with the rest of the limb providing horizontal movement. The theory for a projectile shown in figure 2 is ideal in that the sphere just clears the top of the hurdle. So this presents the minimum path required to carry out the task. Simulations of this movement, using equations (1) and (2), showed that the time taken to project a sphere over the hurdle is considerably shorter than the experimental times. However, applying a constant vertical force during the movement with a smaller impulse of force at the start produced times of about $350 \mathrm{~ms}$, which are typical of the times taken by the participants (figure 8). Adopting constant angular velocity with a vertical force produces a smooth motion but the origins of such smoothness are not clear [22]. Harris and Wolpert propose that precision results in non-jerky movement [23]. Minimizing a quadratic function of the vertical force input and jerk (rate of change of acceleration) is satisfied by a constant vertical force (see the Appendix). While this analysis demonstrates a possible control strategy for producing a constant force, it does not necessarily mean that the physiological system implements this philosophy [24].

During the movement the wrist is flexing or extending but only through a small angle of $10.8^{\circ}$ (balsa) and $11.7^{\circ}$ (aluminium) (figure 7). Hence the wrist supported by the forearm can be considered as providing a constant vertical 


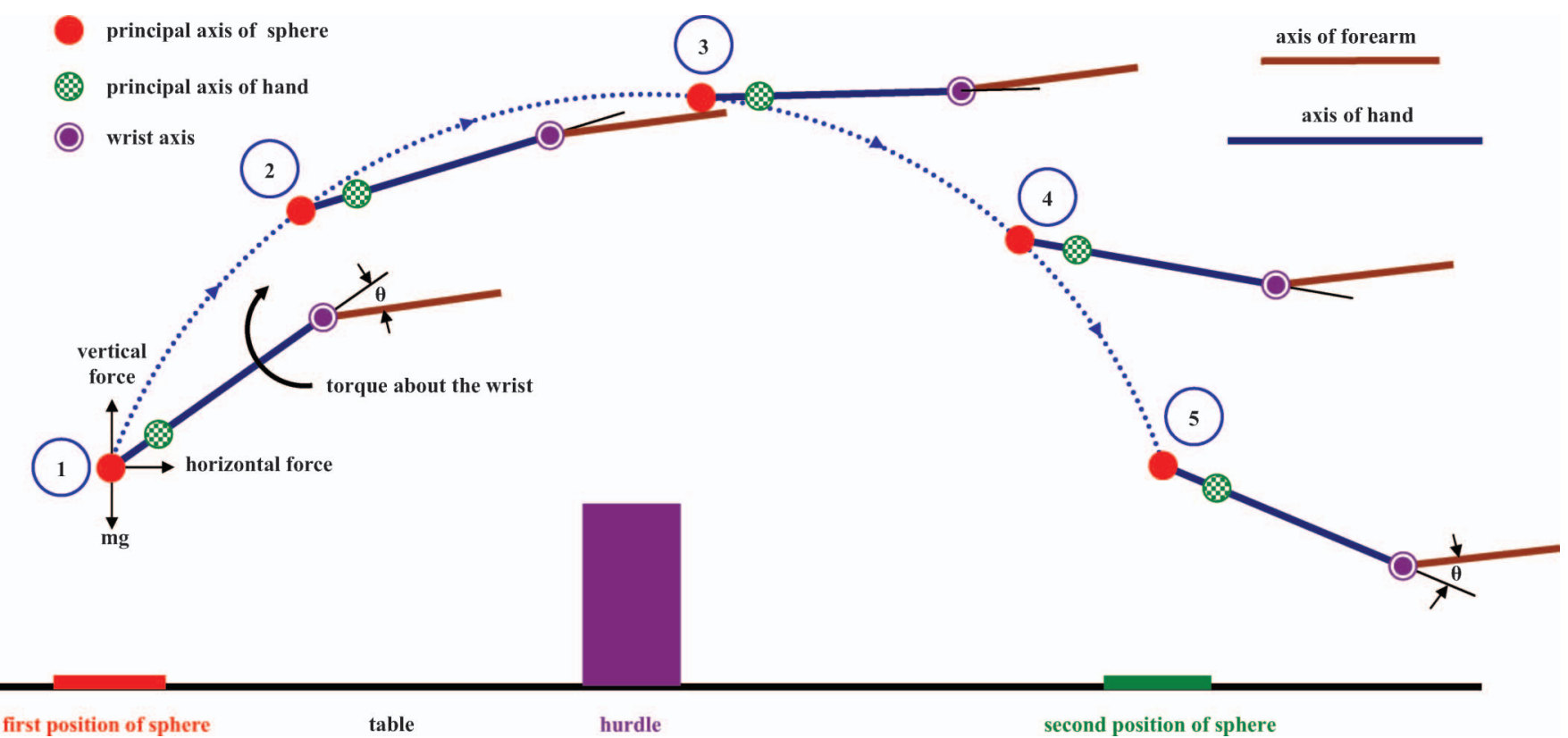

Figure 9. Diagram showing the change in wrist angle, $\theta$, as a sphere is moved over the hurdle from positions 1 to 5 . Included are the forces on the sphere, torque about the wrist and principal axes.

force. The sphere is also being rotated about the wrist but as this is at a constant velocity there is no torque component for this except an impulse at the start of the movement. This control is a 'bang-bang' strategy where the object is rapidly accelerated at the start of a movement, is maintained at constant velocity and decelerated at the end of the movement.

There was no significant difference between the angular velocity developed during the lifting of the balsa sphere and that during the lifting of the aluminium sphere. The neuromuscular control system is therefore maintaining this velocity when the object has negligible mass and when the object has a significant mass (which is about the same mass of a hand). A priori information about the distribution of velocities is used by the central nervous system to achieve targeted movement [24]. Rather than using force feedback from the Golgi tendon organs, the wrist system appears to favour the velocity loop provided by the Type Ia motor neurones. The forearm, humerus and shoulder appear to be using force control to provide a constant vertical force on an object held in the hand.

During the movement of a sphere, there may be radial and ulna deviation which is also carried out typically with a constant velocity. However, this velocity is much smaller than that for flexion and extension, or even zero with a corresponding smaller angular movement. A sphere is moved in the vertical plane with the participant facing the direction of motion (plan view of figure 3) and so the person is in a natural position to use flexion and extension of their wrist. Any abduction or adduction of the wrist results in a horizontal force on the sphere, contributing little or no vertical force, since the hand is in a position of pronation with the palm facing the surface of the table.

\section{Conclusions}

For 10 normal participants, flexion and extension of the wrist is achieved with constant angular velocity when lifting a sphere. There were no significant differences between the means of the angular velocities, absolute velocities, angles or times while lifting a very lightweight sphere and a sphere that had a significant mass. Theoretically the movement of the object follows a classical projectile path with a constant vertical force being maintained by the wrist and upper limb. It is conjectured that the controller for the wrist uses velocity feedback while the rest of a limb maintains a constant vertical force.

\section{Acknowledgements}

The authors wish to thank the Life Sciences Interface Forum at the University of Southampton for their financial support of the research and the participants for their enthusiasm and patience.

\section{References}

[1] Sutherland, D.H., 2002, The evolution of clinical gait analysis Part II. Kinematics, Gait and Posture, 16, 159-179.

[2] Whittle, M.W., 1996, Clinical gait analysis: A review. Human Movement Science, 15, 369-387. 
[3] van Andel, C.J., Wolterbeek, N., Doorenbosch, C.A.M., Veeger, D.H.E.J., and Harlaar, J., 2008, Complete 3D kinematics of upper extremity functional tasks. Gait and Posture, 27, 120-127.

[4] Murgia, A., Kyberd, P.J., Chappell, P.H. and Light, C.M., 2004, Marker placement to describe the wrist movements during activities of daily living in a cyclical task. Clinical Biomechanics, 19, 248-254.

[5] Murgia, A., 2005, A gait analysis approach to the study of upper limb kinematics using activities of daily living. PhD thesis, University of Reading, UK.

[6] Fowler, N.K. and Nicol, A.C., 1999, Measurement of external threedimensional interphalangeal loads applied during activities of daily living. Clinical Biomechanics, 14, 646-652.

-[7] Rau, G., Disselhorst-Klug, C. and Schmidt, R., 2000, Movement biomechanics goes upwards: from the leg to the arm. Journal of Biomechanics, 33, 1207-1216.

-[8] Biryukova, E.V., Roby-Brami, A., Frolov, A.A. and Mokhtari, M., 2000, Kinematics of human arm reconstructed from spatial tracking system recordings. Journal of Biomechanics, 33, 985-995.

[9] Tocheri, M.W., Marzke, M.W., Liu, D., Bae, M., Jones, G.P., Williams, R.C. and Razdan, A., 2003, Functional capabilities of modern and fossil hominid hands: three-dimensional analysis of trapezia. American Journal of Physical Anthropology, 122, 101112.

[10] Leonard, L., Sirkett, D., Mullineux, G., Giddins, G.E.B. and Miles, A.W., 2005, Development of an in-vivo method of wrist joint motion analysis. Clinical Biomechanics, 20, 166-171.

[11] Su, F.-C., Chou, Y.L., Yang, C.S., Lin, G.T. and An, K.N., 2005, Movement of finger joints induced by synergistic wrist motion. Clinical Biomechanics, 20, 491-497.

[12] Rash, G.S., Belliappa, P.P., Wachowiak, M.P., Somia, N.N. and Gupta, A., 1999, A demonstration of the validity of a 3-D video motion analysis method for measuring finger flexion and extension. Journal of Biomechanics, 32, 1337-1341.

[13] Fowler, N.K. and Nicol, A.C., 2001, A three-dimensional biomechanical analysis of the index finger, incorporating measured loading data for selected activities of daily living. Proceedings of the 18th International Society of Biomechanics, Zurich, Switzerland, 8-13 July 2001.

[14] Light, C.M., Chappell, P.H. and Kyberd, P.J., 2002, Establishing a standardized clinical assessment tool of pathologic and prosthetic hand function: normative data, reliability, and validity. Archives of Physical Medicine \& Rehabilitation, 83, 776-783.

[15] Metcalf, C.D., Notley, S.V., Burridge, J.H., Chappell, P.H. and Yule, V.T., 2008, Validation and application of a computational model for wrist and hand movements using surface markers. IEEE Transactions on Biomedical Engineering, 55, 1199-1210.

[16] Metcalf, C.D., 2008, The relationship between movement and function of the wrist and hand: a clinically focused kinematic study. $\mathrm{PhD}$ thesis. University of Southampton.

[17] Zedka, M. and Prochazka, A., 1997, Phasic activity in the human erector spinae during repetitive hand movements. Journal of Physiology, 504, 727-734.

[18] Collins, D.F., Cameron, T., Gillard, D.M. and Prochazka, A., 1998, Muscular sense is attenuated when humans move. Journal of Physiology, 508, 635-643.

[19] MacKinnon, C.D. and Rothwell, J.C., 2000, Time varying changes in corticospinal excitability accompanying the triphasic EMG pattern in humans. Journal of Physiology, 528, 633-645.

[20] Stinear, J.W. and Byblow, W.D., 2002, Disinhibition in the human motor cortex is enhanced by synchronous upper limb movements. Journal of Physiology, 543, 307-316.

[21] Milner, T.E. and Cloutier, C., 1998, Damping of the wrist joint during voluntary movement. Experimental Brain Research, 122, 309-317.

[22] Sejnowski, T.J., 1998, Making smooth moves. Nature, 394, 725-726.

[23] Harris, C.M. and Wolpert, D.M., 1998, Signal-dependent noise determines motor planning. Nature, 394, 780-784.
[24] Körding, K.P. and Wolpert, D.M., 2004, Bayesian integration in sensorimotor learning. Nature, 427, 244-247.

[25] Barnett, S., 1984, Matrices in Control Theory. Krieger Publishing Company, Malabar, FL, USA

\section{Appendix: Optimum control of the vertical force}

A description of optimal control by defining a performance index and Hamiltonian function using Pontryagin's maximum principle can be found in textbooks (e.g. Appendix 4 in [25]).

The vertical equation of motion of a the sphere of mass, $m$ is:

$$
\dddot{y}=\frac{r}{m}-g,
$$

where $r$ is the vertical force (figure 2).

A quadratic performance index, $J$, combining the input vertical force and jerk is

$$
J=\int_{0}^{t_{f}}\left(e^{2}+r^{2}\right) d t
$$

where $\dddot{y}$ is the jerk expressed as:

$$
e=Q \dddot{y}
$$

and $Q$ is a constant.

The Hamiltonian function from these equations is:

$$
H=\left(e^{2}+r^{2}\right)+p\left(\frac{r}{m}-g\right),
$$

where $p$ is the Lagrange multiplier.

Equations (9) and (11) are the associated differential equations, shown below.

$$
\dot{p}=-\frac{\partial H}{\partial e} .
$$

Differentiating equation (8) partially with respect to $e$ :

$$
\begin{aligned}
& \dot{p}=-2 e, \\
& \frac{\partial H}{\partial r}=0 .
\end{aligned}
$$

Differentiating equation (8) partially with respect to $r$ :

$$
2 r+\frac{p}{m}=0
$$

Differentiating equation 12 with respect to time:

$$
2 \dot{r}+\frac{\dot{p}}{m}=0
$$


Eliminating $p$ from equations (10) and (13):

$$
2 \dot{r}-\frac{2 e}{m}=0 \text {. }
$$

Differentiating equation 5 with respect to time:

$$
e=Q \frac{\dot{r}}{m} \text {. }
$$

Eliminating $e$ from equations (14) and (15):

$$
2 \dot{r}-2 Q \frac{\dot{r}}{m^{2}}=0
$$

or

$$
\dot{r}\left(1-\frac{Q}{m^{2}}\right)=0 .
$$

This result implies that $r$ is constant, for an arbitrary value of $m$. An alternative solution is $Q=m^{2}$.

Minimizing a quadratic function of the vertical force input and jerk is satisfied by a constant vertical force input. 